\title{
Logistic Growth for the Nuzi Cuneiform Tablets: Analyzing Family Networks in Ancient Mesopotamia
}

\author{
Sumie Ueda, ${ }^{1}$ Kumi Makino, ${ }^{2}$ Yoshiaki Itoh, ${ }^{1}$ and Takashi Tsuchiya ${ }^{3}$ \\ 1) The Institute of Statistical Mathematics, 10-3 Midori-cho, Tachikawa, Tokyo 190-8562, \\ Japan \\ 2) Kamakura Women's University, 6-1-3 Ofuna Kamakura Kanagawa 247-8512 Japan \\ 3) National Graduate Institute for Policy Studies, 7-22-1 Roppongi Minatoku Tokyo 106-8677 Japan
}

We reconstruct the year of publication of each cuneiform tablet of the Nuzi society in ancient Mesopotamia. The tablets, are on land transaction, marriage, loan, slavery contracts etc. The number of tablets seem to increase by logistic growth until saturation. It may show the dynamics of concentration of lands or other properties into few powerful families in a period of about twenty years. We reconstruct family trees and social networks of Nuzi and estimate the publication years of cuneiform tablets consistently with the trees and networks, formulating least squares problems with linear inequality constraints.

PACS numbers: 89.65.Cd, 89.65.Ef, 02.60.Pn

The observed phase changes of phenomena in human sciences are sometimes well explained by using the models developed in physics. The neolithic transition (i.e., the shift from hunter-gatherer to agricultural economies $)^{\underline{1}}$, change of word ordering rules in natural languages ${ }^{2}$, change of election systems in the present society ${ }^{3}$, and many interesting problems.

The time evolution of Networks based on human interactions in economy, politics, transportation systems, Internet, family trees, etc. is drawing the attention to the present physicists $\underline{4}, \underline{5}$. Here we make use of the interactions in the network of family trees to estimate the years of the contracts given in the Nuzi cuneiform tablets in ancient Mesopotamia to find the simplest nonlinear dynamics, logistic growth of the number of cuneiform tablets.

The Nuzi society $\underline{\underline{6}-\underline{\underline{-}}}$, existed for five or six generations in about 15 th century B.C. 'Nuzi Personal Names' $\underline{9}$ $(\mathrm{NPN})$ is the index for personal names with the kinships for the cuneiform tablets. We introduce the time coordinate of publication of each cuneiform tablet, as well as the birth year and the death year of each person listed in NPN, Fig 2. We formulating least squares problems with linear inequality constraints making use of conditions obtained by kinship relations and other information in NPN. For example we assume that a father is at least 15 years older than his son, contractors were living at the time of the contract, etc. It seems that publication of the cuneiform tablets (documents) are concentrated on a period of twenty years and that they continued to be published until saturation by logistic growth Fig 2 . The large portion of the documents are on land transaction, for example by false adoption given later JEN $208^{7}$. The logistic growth seems to show the dynamics of the emergence of social hierarchy, which is well recognized in philological studies 10.11 , namely the increase of concentration of lands or other properties into few powerful families ${ }^{12}$, as the Tehiptilla family $\underline{13,14}$. The logistic growth seems to be natural to understand the accumulation of cuneiform tablets in Nuzi and support to convince the success of our numerical study. Our time obtained from the least square will reconstruct the life of Tehiptilla and other persons in Nuzi in ancient Mesopotamia.

Nuzi is known for its unique false adoption documents as shown below in Example 1, the cuneiform document JEN 208. The adopted members protected the adoptive father's family in exchange for their properties, such as land estates. There exists a family called Tehiptilla's family, and this family was involved in many contracts and transactions. JEN 208. is the contract between Iluia, a son of Hamattar and Tehiptilla a son of Puhišenni, with the names of witnesses and scribes. The documents JEN are given in ${ }^{7}$, and JENu, given later, are unpublished Nuzi tablets excavated by the Iraq Museum and the American Schools of Oriental Research in 1926.

Example 1. ( $\underline{7,15})$ JEN 208 Adoption tablet. Iluia, a son of Hamattar. He made, Tehiptilla son of Puhišenni, for sonship. Iluia assigned Tehiptilla to as (his) share 2 imer and 3 awehari of land in the large standard, west of the dimtu of Imbi-ili-su, east of the dimtu of eniia. And Tehiptilla [ga]ve to Iluia as his gift 10 imer of barley. If the land ge[ts] a claimant, Iluia shall clear (it), to Te[hip]tilla he (shall) restore (it). The ilku service of the land only [I]luia [shall] bear/ [If Iluia infr]inges (the agreement), [he shall furnish 1 mina of silver (and) 1 mina of gold]. (Rest of the obverse destroyed) L1.17-20:4 seals; some seals destroyed.

[]: broken

(): added by original translator

dimtu: originally means a tower, but also means a region surrounded by city wall, and a district. Jancowska pointed out that there were 71 dimtu in Nuzi ${ }^{16}$

In NPN, the personal names originally written in cuneiform are included as the form of phonetic values with alphabet. For example a name Iluia in JEN 208 is expressed in three cuneiform characters and these phonetic values joined by hyphen as I-lu-ia. Cuneiform character sometimes has more than one phonetic value, and different values may be put by different readers or scholars such as I-lu-ya. We analyzed data only appears in NPN, we could avoid this reading difference. 
Most names found in Nuzi documents are male and inscribed with his father, as Tehiptilla, son of Puhišenni. There are 124 persons named Taia, 7 persons named Iluia, 1 person named Hamattar, etc. We take the sequence of phonetic values listed at first in NPN.

In NPN, individual names are listed in alphabetical order with the information in the original cuneiform documents. Example 2 is the part of NPN associated with two names "Iluia" and "Hamattar" who appear in JEN 208. We see that there is one Hamattar. There are 7 Iluia and some of them represent the same person.

Example 2. Description on Hamattar and Iluia in Nuzi personal names (A syllable is separated by - . The special character $\underline{h}$ is described by $h_{-}\{u\}$. The $\check{s}$ is described by $\mathrm{s}^{\wedge}\{\mathrm{v}\}$.)

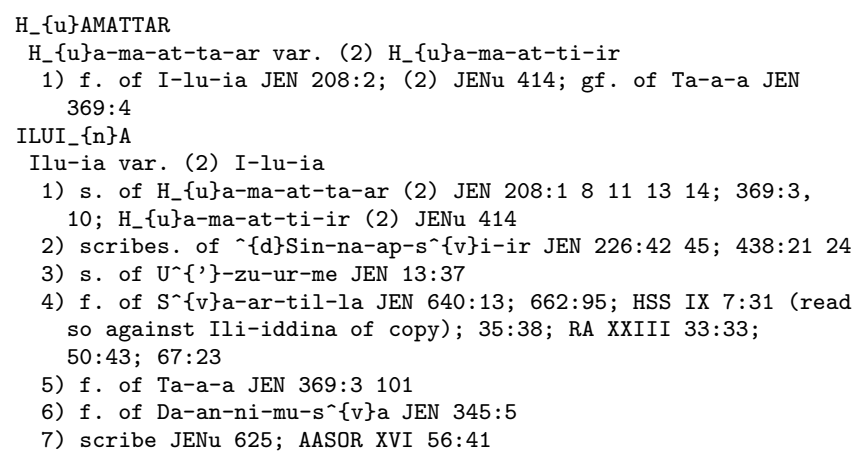

These information include a represented name, the scribal variations, kinship relations, and the line number in which the name appears. For example, JEN 208:2 means that the name appears in the line 2 of the cuneiform tablet JEN 208. In some case, only document is shown without a line number, like JENu 414. The contractor's names are usually included in the first 10 lines, about 2 to 5 lines, in each documents, while the names of witnesses and scribes are given after the first 10 lines.

We list all personal names of JEN 208. JEN 208

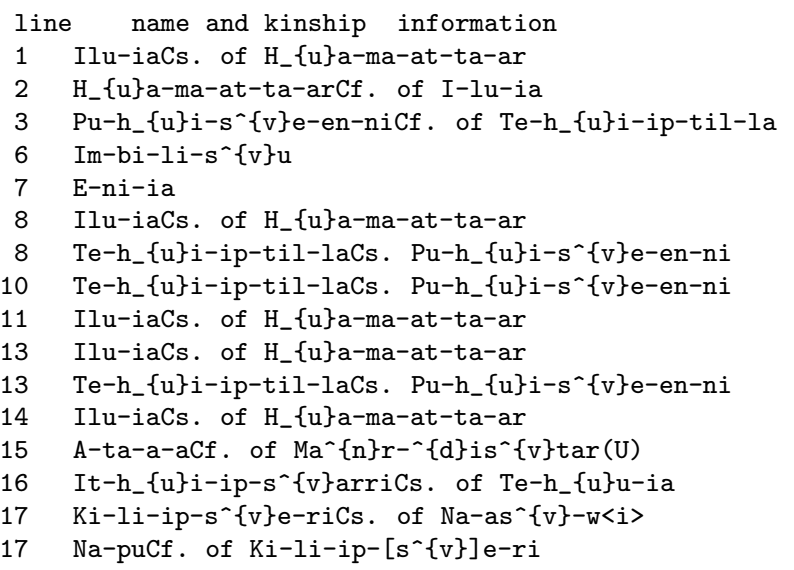

We make a database just from NPN, which is an index of about ten thousands of individuals (about $95 \%$ of them are male) who appear in cuneiform tablets excavated from the site of Nuzi.
Several problem, however, should be solved when constructing a data base from NPN and identify the individuals.

1) Many persons share the same name,

2) Personal names might be written with different cuneiform characters,

3) Some names might be overlapping,

for example, name A can be a single name, but also included in two, three or more generation family trees. These problems were avoided as much as possible by considering other information such as reference numbers and line numbers.

We reconstruct family trees from NPN. We apply the criterion that two persons with the same name are regarded as the same person,

(R1) if the both persons appear on the same line of the same document and regarding them as the same person does not make any contradiction.

or

(R2) if the both persons appear at least in three common documents and their relation is consistent.

For example from the above (R1), we find 1) and 5) of Iluia in Example 2 is the same person. After applying the two criterions (R1) and (R2), we obtained a total of 10343 family trees. We arrange sequentially the family trees obtained directly from the data, as $f_{1}, f_{2}, \ldots, f_{10343}$. Let us say the family tree $A$ and the family tree $B$ are consistent with each other, if and only if at least two names are common in the $A$ and the $B$ and there is no contradiction in their kinship relations for the names both in the $A$ and the $B$. Starting from the initial set of the 10343 family trees, we apply the sequential algorithm recursively to unify mutually consistent family trees. We continue the procedure until there is no family tree to be unified ${ }^{17,18}$ and get family trees as shown in Fig 1, whch is a part of 16 members obtained by our procedure. The Tehiptilla family tree estimated by our sequential method, using just NPN, is about the half size of the one ${ }^{13,14}$, constructed by using all possible information in contents of cuneiform tablets, and does not coincide with it in part.

We obtained 1 family tree with 6 generations 16 members, which is the largest family tree obtained, the Tehiptilla family tree given in part in Fig 1, 2 family trees with 5 generations, one is with 5 members and the other is with 11 members, 3 family trees with 4 generations 4 members, and 61 family trees with 3 generations. 4096 family trees with 2 generations and family trees and 1987 single family trees and 24 family trees of one generation with size 2 . Here we note that most of the 2 generation family trees are pairs of a father and his son, and a single family tree means a person without any given kinship in NPN. The Tehiptilla family existed for 6 generations. If the two personal names appear in a tablet, we assume the two persons lived in the same period. Based on this idea, we associate each individuals to one of the six generations of the Tehiptilla family.

Consider the family of Hamattar, in Example 1, 2, of three generations. Iluia, the son of Hamattar, is in the 


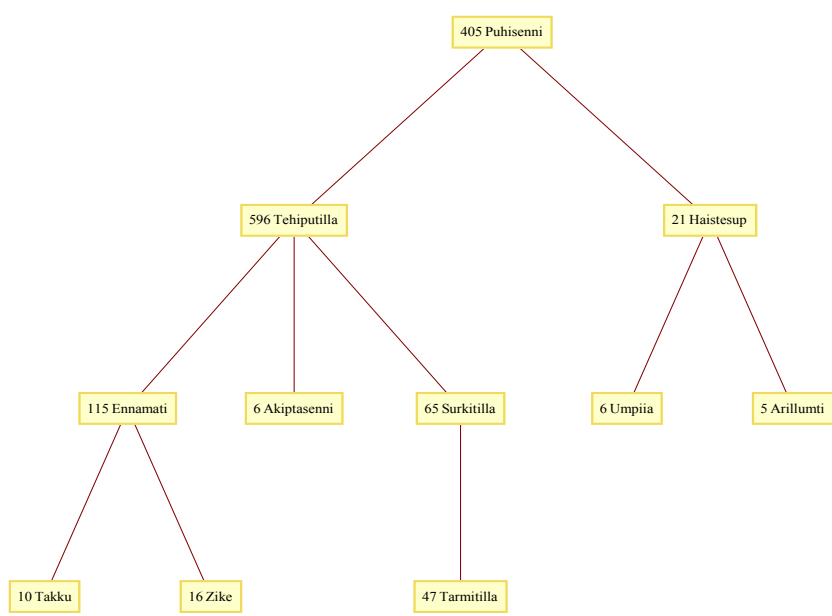

FIG. 1. Tehiptilla and his father, brother, son, and grandson. The number of appearance of each name in documents is shown as 596Tehiptilla. The birth year and death year of each name ${ }^{12}$, in the bracket () is as, Puhišenni (39.06- 99.06), Tehiptilla (59.06-114.06), Haištešup (63.27114.06), Akiptašenni (74.35-134.27), Ennamati (74.06114.06), Šurkitilla (74.07-105.69), Arillumti (78.27-135.74), Umpiia(78.27-114.06 ), Takku (89.06-139.78), Zike (89.06116.70), Tarmitilla (89.07-137.56).

tablets JEN 208 and JEN 369. Taia, the grand son of Hamattar, is in just JEN 369. Puhišenni Tehiptilla the son of Puhišenni and Enna-mati the son of Tehiptilla are in the tablets JEN 208, JEN 369, and JENu 414. Other personal names of the Tehiptilla family are not in the tablets. So let Puhišenni be the first generation, and Tehiptilla be the second generation. Hamattar is approximately considered to be in the first generation, Iluia in the second generation and Taia in the third generation. So we can see approximate correspondences of generation among family trees.

We observe that most of the names in NPN are in one person family tree or the two person family tree $\underline{18}$. They may be considered as ordinary people in obscurity. They appear in the cuneiform tablets only in the earlier period of the logistic growth Fig 2. After the contracts they do not appear in cuneiform tablets. $45 \%$ of them share documents with Tehiptilla family indicates that the Tehiptilla family occupied the high rank of the Nuzi society. Through the false adoption contracts, ownership of land estates and other properties of them were passed to the hands of Tehiptilla family. Several other high ranked families were also observed. 17 families with 79 people shares documents with $70 \%$. The results show the social hierarchy in the city of Nuzi11.

The dynamical system, for the logistic growth,

$$
\frac{d}{d t} f(t)=\frac{1}{\beta}(1-f(t)) f(t)
$$

has the solution $f(t)=1 /\left(1+e^{-\frac{t-\mu}{\beta}}\right)$. The system finds applications in biology, demography, economics, chemistry, sociology, political science, and many other fields.
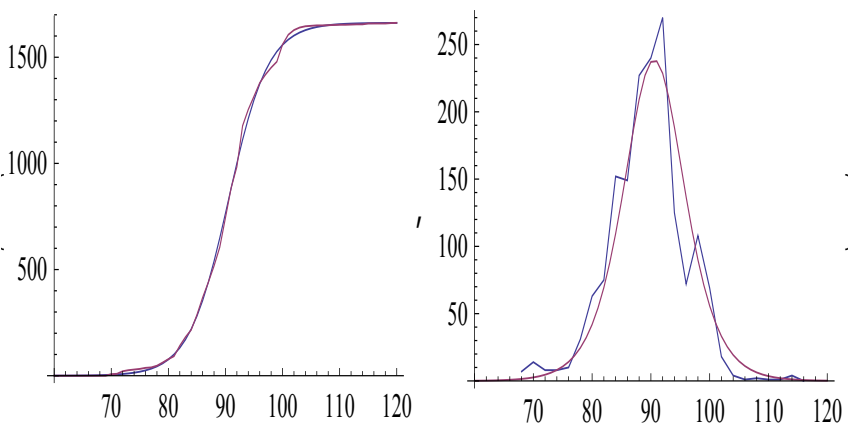

FIG. 2. Data (publication years obtained by mathematical optimization) and logistic growth. Left figure: data and estimated logistic distribution function. Right figure: data and estimated logistic probability density. The value of the maximum log likelihood for the logistic distribution is -5404.04 , while that of normal distribution is -5438.27 .

For example in population genetics the relative abundance of a advantageous mutant gene increases in a population until saturation $f(t)=1$. Assuming the above dynamical system for the ratio $f(t)$ of properties owned by powerful families as the Tehiptilla family, we have the probability density of logistic distribution function with mean $\mu$ and scale parameter $\beta$,

$$
\frac{e^{-\frac{t-\mu}{\beta}}}{\beta\left(1+e^{-\frac{t-\mu}{\beta}}\right)^{2}} .
$$

which seems to explain the distribution of publication years of the cuneiform tablets Fig 2. We can imagine the land owner class appeared through the contracts, as in JEN 208 of Example 1, during the period and after the period the society changes to another stage of social hierarchy in which lands are owned by few families.

Now we show the outline of our optimization method how we estimate the the publication year of each document, birth year and the death year of each individual, consistently with NPN. As we easily imagine NPN alone is not enough to restore a detailed scenario of population dynamics of society. The index just contains (partial) kinship information and about those people who appear in a document simultaneously, that is to say, were alive at the time of contract. To fill this gap, we make use of a reasonable prior information and employ a simulation and optimization approach.

First we give an initial lifespan to each person uniformly at random on the interval $[20,60]$ independently. Based on this given initial set of lifespans of people, we formulate an optimization problem to find a configuration of birth year, death year of individuals and publication year of cuneiform tablets consistent with the information from NPN. Through solving the optimization problem, we obtain a configuration which is closest to the initial lifespan among all configurations consistent with the document information. 
Though the obtained configuration is different depending on the initial lifespan given at random. We find a common social structure among the obtained configurations which would reflect what was going on in ancient Nuzi.

We formulate the optimization problem for unknown variables, the birth year $b_{i}$ and the death year $d_{i}$ of the person $i$, the id number of the father $f_{i}$ and the mother $m_{i}$ of the person $i$. and the published year $P_{k}$ of the document $k$. The quadratic optimization problem to minimize $\sum_{i}\left(d_{i}-b_{i}-\mu_{i}\right)^{2}$ finds a configuration, whose lifespans are closest to the initial lifespans $\mu_{i}$, in the least square sense, subject to that

(I) $b_{i} \geq b_{f_{i}}+g_{f}$, ( a male becomes a father elder than $g_{f}$ years old for all person $i$ whose father appear in NPN), and $d_{f_{i}} \geq b_{i}, b_{i} \geq b_{m_{i}}+g_{m}$ (a female becomes a mother elder than $g_{m}$ years old, for all person $i$ whose mother appear in NPN), and $d_{m_{i}} \geq b_{i}$.

(II) $P_{k} \geq b_{i}+g_{p}, \quad d_{i} \geq P_{k}$ ( a person appeared in a document is supposed to be greater than $g_{p}$ years old for all documents $k$ and person $i$ in the document $k$, and $i$ lived at the time of contract ),

(III) $P_{906}=100$ to fix the origin of coordinate, where 906 is the id for JEN 525. JEN 525 contains 52 different individual names. We make a set of names, each of which is connected with directly or indirectly to one of the member through kinship relation or common contracts, and call it extended members of JEN 525. For each document we make extended members. JEN 525 has the largest extended members among all documents which includes Tehiptilla's family.

The above least square problem was solved with a software package NUOPT (Mathematical Systems Inc.). The optimization result is depending on the initial set of lifespans. We conducted the computation for Fig 2 assuming $g_{f}=15, g_{m}=20$, and $g_{p}=10$.

The result of our reconstructing family tree makes the number of variables and constraints smaller, as we find 1) and 5) of Iluia in ex.2 is the same person. A total of 10060 persons appears in our study of NPN. We denote by $\mu_{i}$ the lifespan of the person $i$, for $i=1, \ldots, 10060$. The number of documents is 1662 .

The convex quadratic program with $21782(=10060 \times$ 2 (representing $\left.b_{i}, d_{i}\right)+1662\left(\right.$ representing $\left.P_{k}\right)$ ) variables. We have 39357 constraints. The above (I) makes $4241 \times 2$ constraints of them, (II) makes $15437 \times 2$ constraints of them, and (III) makes 1 constraint of them.

We carried out the optimization 10 times independently, changing the initial span given at random. The structure of the optimal solutions are more or less the same regarding to the properties we reported here.

\section{ACKNOWLEDGMENTS}

This work is in part supported by Ministry of Education Science and Culture of Japan ( Research Project 07207245, and Research Project 09204245), and ISM Cooperative Research (2011- ISMCRP -1022). Y. I. is supported in part by US National Science Foundation Grant DMS 0443803 to Rockefeller University.

${ }^{1}$ J. Fort, and V. Mendez, Time-Delayed Theory of the Neolithic Transition in Europe, Physical Review Letters, 82, 867-870 (1999).

${ }^{2}$ Y. Itoh, and S. Ueda, The Ising model for changes in word ordering rules in natural languages, Physica D, 198, 333-339 (2004).

${ }^{3} \mathrm{Y}$. Itoh, and S. Ueda, Observed phase changes and the random packing model for elections in Japan, Physica D 142, 29-40 (2000).

${ }^{4}$ P. L. Krapivsky, S. Redner, and F. Leyvraz, Connectivity of growing random networks, Physical Review Letters, 85, 46294532 (2000).

${ }^{5}$ Y. Itoh, C. Mallows, and L. Shepp, Explicit sufficient invariants for an interacting particle system, Journal of Applied Probability. 35, 633-641(1998).

${ }^{6}$ R. F. S. Starr, Nuzi; report on the excavation at Yorgan Tepa near Kirkuk, Iraq, conducted by Harvard University in conjunction with the American Schools of Oriental Research and the University Museum of Philadelphia, 1927-1931, Volume 2, Plates and Plans, Harvard University Press (1937).

${ }^{7}$ E. Chiera, I. J. Gelb, and E. R. Lacheman, Joint expedition with the Iraq Museum at Nuzi, American Schools of Oriental Research; Publications of the Baghdad School, (P. Geuthner, Paris, 1934).

${ }^{8}$ M. A. Morrison, and D. I. Owen, Studies on the Civilization and Culture of Nuzi and the Hurrians in Honor of Ernest R. Lacheman on his Seventy-First Birthday, April 29, 1981, ( Eisenbrauns, Winona Lake, Indiana, 1981).

${ }^{9}$ I. J. Gelb, P. M. Purves, and A. A. MacRae, Nuzi personal names (The University of Chicago Press, Chicago Illinois, 1943).

${ }^{10}$ N. B. Jankowska, Communal Self-Government and the king of the state of Arrapha, Journal of the Economic and Sosial History of the Orient 12 (3), 233-282 (1969).

${ }^{11}$ H. Lewy, The Nuzian feudal system, Orientalia 11, 319-329 (1942).

${ }^{12}$ S. Ueda, Statistical Mathematics Approach to Human Sciences, (Phd Thesis, Graduate University for Advanced Studies, 2010) (in Japanese).

${ }^{13}$ M. A. Maidman, The Tehiptilla family of Nuzi - a genealogical reconstruction-, Journal of Cuneiform Studies 28 (3), 127-155 (1976).

${ }^{14}$ M. A. Maidman, Nuzi texts and their uses as historical evidence ( Society of Biblical Literature, Atlanta, 2010).

${ }^{15} \mathrm{~K}$. Makino, Social change reflected in the source of false adoption contracts at Nuzi. Shigaku 60(1), 91-119 (1991) (in Japanese with English title).

${ }^{16}$ N. B. Jancowska, Extended family commune and civil government in Arrapha in the fifteenth to fourteenth century B.C. pp. 235-256. in I. M. Diaconoff ed. Ancient Mesopotamia: SocioEconomic History. (A Collection of Studies by Soviet Scholars, Moscow, 1969).

${ }^{17}$ Y. Itoh, M. Ishiguro, S. Ueda, and K. Makino, Estimating population from the kinship data in an ancient society (CDROM), Report of Research Grant 09204245, Ministry of Education, Science and Culture of Japan, 1998) (in Japanese).

${ }^{18} \mathrm{~S}$. Ueda, K. Makino, and Y. Itoh, Reconstructing family trees in ancient population from the Nuzi personal names, Proceedings of the Institute of Statistical Mathematics 53, 285-295 (2005) (in Japanese with English summary). 